Meeting report

\title{
Advances in the genetics and epigenetics of gene regulation and human disease Kristine Kleivi
}

Addresses: Medical Biotechnology, VTT Technical Research Center of Finland, 20521 Turku, Finland and Department of Genetics, Institute for Cancer Research, Rikshospitalet-Radiumhospitalet Medical Center, o310 Oslo, Norway. Email: kristine.kleivi@vtt.fi

Published: 24 August 2006

Genome Biology 2006, 7:325 (doi:10.1 186/gb-2006-7-8-325)

The electronic version of this article is the complete one and can be found online at http://genomebiology.com/2006/7/8/325

(C) 2006 BioMed Central Ltd

A report on the Human Genome Organisation (HUGO) II th Human Genome Meeting, Helsinki, Finland, 3I May-3 June 2006.

At the recent annual meeting on the human genome in Helsinki, organized by the Human Genome Organisation (HUGO), close to 700 scientists gathered to present and discuss the latest advances in genome research. This report presents some selected highlights.

\section{Genome variation, gene expression and disease susceptibility}

Through their effects on gene expression, polymorphisms in the human genome can contribute to phenotypic variation and disease susceptibility. For many diseases, such as cancer, great effort is being made to study the sequence variants that contribute to disease susceptibility. The impact of genetic variation on common diseases was addressed by Kari Stefansson (deCODE Genetics, Reykjavik, Iceland), who gave an update on the identified sequence variants that may increase the risk of developing type 2 diabetes, prostate cancer, myocardial infarction, stroke and schizophrenia. In the past decades, type 2 diabetes has become a major health problem in the Western world, as both its incidence and its prevalence have increased rapidly. Stefansson reported his group's recent discovery of an inherited variant of the gene $T C F 7 L 7$, encoding a protein called transcription factor 7-like 2 located on chromosome 10, which is estimated to account for about $20 \%$ of the diabetes cases. They have also showed an association between a common genetic variant in the microsatellite DG8S737 at chromosome band 8q24, which may contribute to the development of prostate cancers in European and African populations.

Single-nucleotide polymorphism (SNP) genotypes correlated with gene-expression data in breast tumors were presented by Vessela Kristensen (The Norwegian Radium Hospital,
Oslo, Norway). For genotyping, she and her colleagues selected sets of genes involved in reactive oxygen species signaling (ROS) and the repair of DNA damage caused by ROS that is, pathways that are generally affected by chemotherapy and radiation therapy. Using various statistical approaches, the genetic association between SNPs in genes involved in the ROS pathways and the expression levels of mRNA transcripts from a panel of breast cancer patients were assessed. Regulatory SNPs in the genes EGF, IL1A, MAPK8, XPC, SOD2 and $A L O X 12$ were associated with alterations in the expression levels of several transcripts. Kristensen also showed that a set of SNPs were linked to a cluster of transcripts participating in the same functional pathway.

Thomas Hudson (McGill University, Montreal, Canada) described several resources and technologies that are available to study the impact of genome variation on gene expression. He and his colleagues systematically studied a subset of genes whose alleles show large differences in expression in lymphoblastoid cell lines. These data were integrated with HapMap data to search for haplotypes associated with mRNA expression at flanking genes. Hudson described the discovery of 16 loci harboring a common haplotype affecting the total expression of a gene, that is, all the alleles of the gene, and of 17 loci that affected relative allelic expression in heterozygous samples. To better understand the mechanisms controlling this gene expression, cis-acting polymorphisms need to be studied in the human genome in larger sample sets and tissue panels.

\section{DNA methylation and epigenetic modification of the genome}

Methylation of $\mathrm{CpG}$ islands has an important role in controlling gene expression during mammalian development, and is frequently altered in diseases such as cancer. DNA methylation was extensively discussed in the meeting. For example, Carmen Sapienza (Temple University Medical School, 
Philadelphia, USA) reported that imprinted regions in humans are historical hotspots of recombination. Together with specific DNA sequences, epigenetic factors may have an important influence on the rate of meiotic recombination and the position of cross-overs. Using in silico and in vitro analyses, Sapienza's group have shown a relationship between increased rates of meiotic recombination and genomic imprinting. Imprinted regions showed more linkage disequilibrium, and had a significantly higher number of small haplotype blocks, than the non-imprinted regions. Their findings suggest that several factors, including both specific DNA sequences and epigenetics, are involved in controlling meiotic recombination in humans.

Nutritional influences during prenatal and early postnatal development may affect gene expression, and subsequently the phenotype, through epigenetic gene regulatory mechanisms. Nutrition is important in providing methyl donors for DNA, and some genes are especially sensitive to nutritional changes during embryogenesis. Rob Waterland (Baylor Collage of Medicine, Houston, USA) has used mouse models to show that some alleles are particularly susceptible to changes in methylation due to maternal nutrition. For example, supplementary nutrition can lead to increasing body weight across several generations of offspring. He and his colleagues postulate that maternal nutrition before and during pregnancy may affect the establishment of $\mathrm{CpG}$ methylation and the life-long expression of metastable epialleles (epigenetically modified alleles) in humans.

Whereas the majority of $\mathrm{CpG}$ islands in the genome are normally unmethylated, a sizeable fraction is prone to methylation in various cell types and pathological conditions. Christoph Bock (Max Planck Institute for Informatics, Saarbrücken, Germany) described his group's work predicting CpG methylation on the basis of DNA sequence and genomic location. Using a bioinformatics approach, they were able to distinguish $\mathrm{CpG}$ islands that are prone to methylation from those that are not. For example, on chromosome 21, they were able to predict the $\mathrm{CpG}$ island methylation rate with $90 \%$ accuracy, which was later confirmed by in vitro analyses. This study revealed that the DNA composition of $\mathrm{CpG}$ islands, the sequence, the structure and the number of repeats play an important role in predisposing CpG islands to DNA methylation. Furthermore, these features can also be used to predict the $\mathrm{CpG}$ methylation pattern of the whole genome.

\section{Regulatory genomics}

Knowledge about transcription factors, their binding specificities and the assembly of their binding sites to form tissuespecific enhancer elements is critical for understanding key regulatory mechanisms of human gene expression. Outi Hallikas (University of Helsinki, Finland) aimed to determine the binding specificities of transcription factors that are involved in growth control, and to find evolutionarily conserved enhancer elements that drive organ-specific expression of genes that regulate a cell's progression through the cell cycle. By using a high-throughput method for determining transcription factor binding sites, Hallikas reported the binding sites of the transcription factors GLIs, TCF4 and c-ETS1, which are involved in different signaling pathways such as those leading from the signal protein Wnt and the Ras/MAPK intracellular signaling module. To identify the mammalian enhancer elements, Hallikas and colleagues have developed a new computational tool (Enhancer Element Locator; available online [http://www.cs.helsinki.fi/u/kpalin/EEL/]), and used it to predict active transcription factor binding sites. Validation of these in transgenic mice revealed the presence of enhancers in c-Myc and N-Myc, genes that play a role in growth control and tumorigenesis.

From the same group, Mikael Björklund presented a genome-wide RNA interference (RNAi) analysis of genes that are involved in cell-cycle control and cell-size regulation in Drosophila. Using flow cytometry, Björklund and colleagues analyzed the RNAi-induced loss-of-function of $70 \%$ of the genes in Drosophila, including those conserved in humans, on cell-cycle progression by flow cytometry. Genes controlling several cellular processes were identified, including cell size, cytokinesis, apoptosis and phases of the cell cycle. In addition, a translational regulator (eIF-3p66) associated with the cyclin/cyclin-dependent kinase pathway was identified.

The combination of RNAi and gene-expression profiling provides further insight into gene function and the regulatory networks controlling expression. Ilaria Piccini (Max Planck Institute for Molecular Genetics, Berlin, Germany) presented their genome-wide analysis of transcription factors in association with gene regulation. Using RNAi, Piccini and colleagues are knocking down the expression of 200 transcription factors involved in human developmental processes in a panel of cell lines, and investigating the downstream targets of these at the transcriptome level. They have initially focused on transcription factors encoded by chromosome 21, and as an example, Piccini described the identification of 72 potential target genes, containing both known and novel targets, that were dysregulated when expression of the transcription factor gene $B A C H 1$ was silenced.

\section{DNA amplifications in cancer genomes}

Gene amplifications are seen in a variety of human cancers, and are often associated with poor clinical outcome for the patient. Target genes for amplified regions are often oncogenes, which may be used as therapeutic, prognostic and diagnostic targets. Therefore, increasing the knowledge of DNA copy number amplifications in human neoplasms is important. This issue was addressed by Samuel Myllykangas (University of Helsinki, Finland) who presented an extensive analysis of DNA copy number amplifications in human 
cancers. Using data from published comparative genomic hybridization studies, they performed an in silico analysis of DNA copy number changes in approximately 4,500 samples from over 70 different neoplasms. Computational analysis identified the different amplification hotspots, which were spread over large parts of the genome and frequently colocalized with known fragile sites, cancer genes and virus integration sites. Amplification of some chromosomal regions was observed in the majority of the cancers studied, whereas other amplifications were cancer-site specific. From the characteristic amplification profiles, Myllykangas showed that cancers with similar cellular origin and histology, such as breast and prostate adenocarcinomas, clustered together. Their discoveries show the relevance of global studies on DNA amplifications in human cancers, and suggest diagnostic and predictive possibilities.

The annual Human Genome Meeting of 2006 was an inspiring meeting, updating us on the latest knowledge in human genomics. The power of combining high-throughput experimental approaches with genome-wide bioinformatics, systems biology and data integration was emphasized. Thus, it was a successful demonstration of strategies that will be increasingly useful in human genetics in the future. 